\title{
Effects of Chitin and Chitosan on Collagen Synthesis in Wound Healing
}

\author{
Kazuo KOJIMA ${ }^{1)}$, Yoshiharu OKAMOTO ${ }^{1)}$, Kazuya KOJIMA ${ }^{2}$, Katsuyuki MIYATAKE $^{1)}$, Hiroshi FUJISE²), \\ Yoshihiro SHIGEMASA ${ }^{3)}$ and Saburo MINAMI ${ }^{1)}$
}

${ }^{1)}$ Department of Veterinary Surgery, Faculty of Agriculture, Tottori University, Tottori 680-8553, ${ }^{2)}$ Department of Veterinary Pathology II, Azabu University, School of Veterinary Medicine, Fuchinobe, Kanagawa 229-8501 and ${ }^{3)}$ Department of Materials Science, Faculty of Engineering, Tottori University, Tottori 680-8552, Japan

(Received 15 January 2004/Accepted 27 July 2004)

\begin{abstract}
Collagen synthesis was evaluated by measuring prolyl hydroxylase (PHL) activity induced within rat granulation tissue by a polyester non-woven fabric (NWF, $1 \times 1 \mathrm{~cm}, 0.6 \mathrm{~mm}$ in thickness) impregnated with a chitin or chitosan suspension ranging in concentration from 0.1 to $10 \mathrm{mg} / \mathrm{m} l$. In addition, PHL activity induced in rat granulation tissue by a NWF impregnated with a phosphate buffer solution was examined as a control. The PHL activity in each group remained low until 4 days post-implantation (Day 4). However, in the $10 \mathrm{mg}$ chitin group, the PHL activity increased rapidly without scatter of the data at Day 7 and remained at a plateau until Day 14. In other groups, PHL activity increased linearly until Day 14. The data varied widely at Day 7 . Compared to chitosan, chitin at the higher concentration was found to induce stable collagen synthesis in the early wound healing process.

KEY WORDS: chitin, chitosan, prolyl hydroxylase.
\end{abstract}

J. Vet. Med. Sci. 66(12): 1595-1598, 2004

Chitin and chitosan have been widely studied in both engineering and medicine. In the field of medicine, the effects of chitin and chitosan on wound healing have been the focus of great attention since Prudden et al. published a paper in 1970 stating that these agents accelerate wound healing [15]. Numerous reports describe the stimulatory effects of chitin and chitosan on tissue reactions involved in wound healing [1, 3-5, 7-13]. Their most notable property is their propensity to induce abundant granulation tissue formation with angiogenesis. Previous reports indicated that chitin and chitosan induced fine collagen fibers histologically $[3,11]$. Moreover, chitin was found to enhance synthesis of types I, III, and IV collagen [4]. However, these reports indicate only the properties of the collagen synthesized. To understand more about wound healing acceleration by chitin and chitosan, it is important to clarify the quantity of collagen synthesized as well as its properties. In this paper, we evaluated the degree to which chitin and chitosan stimulate collagen synthesis by measuring prolyl hydroxylase activity, an activity necessary for collagen synthesis in rats [18].

Thirty Wistar rats (adult females, weighing 270-300 g) were used in this study. The animals were purchased from Hamaguti Laboratory Co., Ltd. (Kobe, Japan). Chitin and chitosan were supplied by Sunfive Co., Ltd. (Tottori, Japan). Molecular weights and rates of deacetylation were determined using the viscoanetric method [19] and infra-red spectroscopic method [17], respectively. The data obtained for chitin and chitosan yielded molecular weights of 300 and $80 \mathrm{kD}$, and deacetylation rates of $<10$ and $>80 \%$, respectively. Chitin and chitosan having a mean particle size of $3.5 \mu \mathrm{m}$ were sterilized separately using ethylene oxide gas and suspended in phosphate buffered solution (PBS, pH 7.2) at a concentration of $10 \mathrm{mg} / \mathrm{ml}$. According to our measurements in a previous investigation [5], the optimum doses of chitin and chitosan in granulation tissue formation in rats were 1.0 and $10 \mathrm{mg} / \mathrm{ml}$, and 0.1 and $1.0 \mathrm{mg} / \mathrm{ml}$, respectively. In the chitin and chitosan at the higher concentrations, excessive inflammatory reactions occurred. Therefore, the chitin and chitosan suspensions were adjusted with PBS to final concentrations ranging from 1.0 and $10 \mathrm{mg} / \mathrm{ml}$, and 0.1 and $1.0 \mathrm{mg} / \mathrm{ml}$, respectively.

A polyester non-woven fabric (NWF: Sontara 8100, Dupont, U.S.A., $1.0 \times 1.0 \mathrm{~cm}, 0.6 \mathrm{~mm}$ in thickness) was sterilized by ethylene oxide gas and then impregnated with $0.1 \mathrm{~m} l$ of a chitin $(1.0$ and $10 \mathrm{mg} / \mathrm{m} l)$ or chitosan $(0.1$ and 1.0 $\mathrm{mg} / \mathrm{ml}$ ) suspension. As a control, NWF was impregnated with $0.1 \mathrm{~m} l$ of PBS alone.

The rats were divided into one chitin group and one chitosan group, each comprised of 15 rats divided into three subgroup of 5 rats. After anesthesia was induced via an intramuscular injection of atropine sulfate $(0.05 \mathrm{mg} / \mathrm{kg})$, ketamine- $\mathrm{HCl}(15 \mathrm{mg} / \mathrm{kg})$, and propionyl promazine $(0.05$ $\mathrm{mg} / \mathrm{kg}$ ), the dorsal supracostal region of each rat was shaved and disinfected with chlorhexidine gluconate. A straight full-thickness skin incision of $10 \mathrm{~cm}$ was made over the dorsal midline. Five rats belonging to the chitin group (the first subgroup) received the NWFs impregnated with the $1.0 \mathrm{mg}$ chitin suspension, the $10 \mathrm{mg}$ chitin suspension, and the PBS at their cranial, middle, and caudal areas, respectively. Two implants were placed separately on each side of the dorsal midline of each area, at a distance of $2 \mathrm{~cm}$ apart. In the second subgroup of the chitin group, the three different NWFs were placed around the middle, caudal, and cranial areas, respectively, and in the third subgroup they were placed near the caudal, cranial, and middle areas, respectively. The same arrangement was adopted for the chitosan group. The implants were fixed at each corner by interrupted sutures with 3-0 nylon, then the skin was closed by interrupted sutures. 
At 2, 4, 7, 11, and 14 days post-implantation, one rat from each subgroup of the chitin and chitosan groups was randomly selected and euthanized by excessive ether anesthesia. The implants were then removed from the wounds and was divided into 2 pieces; one for the measurement of prolyl hydroxylase (PHL) activity and the other for histological observation. If bacterial infection of an implant was suspected, the sample was eliminated from the following measurement. The number of samples ranged from 3 to 5 in each group. One piece of each implant was minced with scissors. Three $\mathrm{m} l$ of $0.25 \mathrm{M}$ sucrose solution was added to $1 \mathrm{~g}$ of each wet implant, a homogenizer was used to obtain a tissue solution, the solution was centrifuged $(15,000 \times \mathrm{g}, 15$ $\min$, at $4{ }^{\circ} \mathrm{C}$ ), and the supernatant was obtained for enzyme assay.

The required PHL substrate and the enzyme assay were performed according to Hutton et al. [2]. Thus, developing chick eggs were cultivated in the presence of $\left[{ }^{3} \mathrm{H}\right]$ - labeled prolyl, a collagen precursor with $\left[{ }^{3} \mathrm{H}\right]-\mathrm{H}_{2} \mathrm{O}$ liberated with the action of PHL was distilled, and the radioactivity was measured. The level of enzyme activity noted was expressed as radioactivity ( $\mathrm{dpm} / \mu \mathrm{g}$ protein). The remaining piece of each implant was fixed in $10 \%$ formalin, and paraffin sections were prepared and stained with hematoxylin and eosin (H\&E).

Analysis of variance was performed by the Student's unpaired $t$-test. Differences were considered significant at $p<0.05$.

Changes in activities of PHL per $\mu$ g-protein are shown in Fig. 1. In the control group (NWF alone), its activity was very low until 4 days post-implantation (Day 4), after which its activity increased linearly. Activities noted at Day 7, 11, and 14 were 5.5-, 8-, and 9.5-fold compared to that of Day 4. At Day 7, each data varied widely (ranging from 1.9 to $7.9 \mathrm{dpm} / \mu \mathrm{g}$-protein). In the chitin group, the change in PHL activity at a concentration of $1.0 \mathrm{mg} / \mathrm{m} l$ was similar to that of the control, though scatter of the data was seen at Day 7 (ranging from 1.1 to $8.2 \mathrm{dpm} / \mu \mathrm{g}$-protein). On the other hand, PHL activity in the chitin group at a concentration of $10 \mathrm{mg} / \mathrm{ml}$ increased rapidly at Day 7 (8.5-fold compared to that of Day 4) and then remained at a plateau until Day 14. Scatter of the data was not seen at Day 7 (ranging from 7.7 to $9.0 \mathrm{dpm} / \mu$ g-protein). At Day 7 , the activity of PHL was highest in the $10 \mathrm{mg}$ chitin group in comparison with other groups, but this difference was not significant. In the chitosan group, changes in PHL activity at concentrations of 0.1 and $1.0 \mathrm{mg} / \mathrm{m} l$ were similar to that of control, though scatter of the data was seen at Day 7 (ranging from 1.6 to $8.5 \mathrm{dpm} /$ $\mu \mathrm{g}$-protein in the $0.1 \mathrm{mg}$ chitosan group and from 1.7 to 7.5 $\mathrm{dpm} / \mu \mathrm{g}$-protein in the $1.0 \mathrm{mg}$ chitosan group, respectively). At Day 14, the PHL activity was higher in the chitosan group in comparison with other groups, but this difference was not significant. Changes in PHL activity per wet implant with time were also similar to that of its activity per $\mu$ g-protein. There was no relationship between PHL and the site impregnated in each group.

Histological findings at Day 2 revealed a small number of
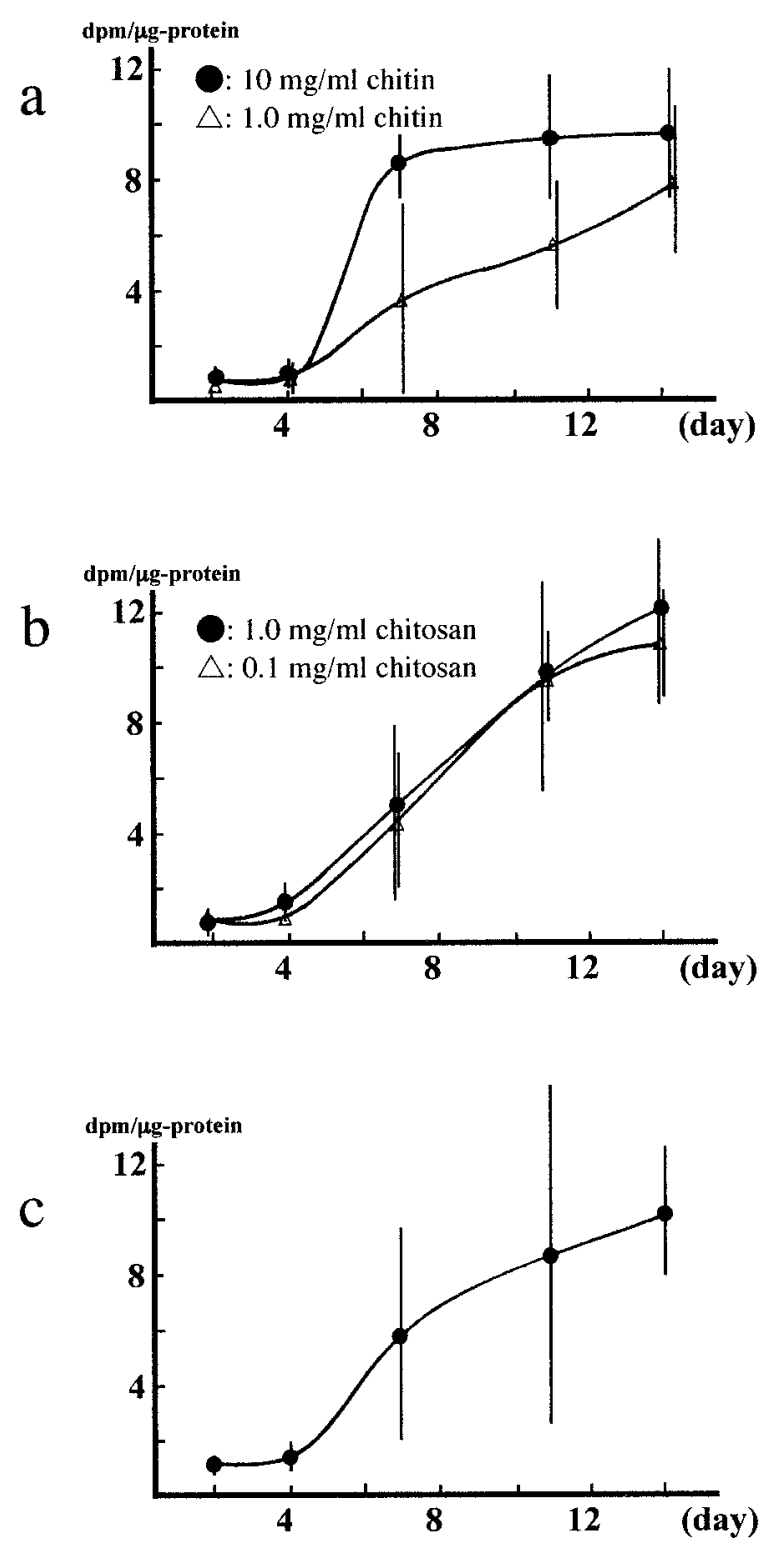

Fig. 1. Prolyl hydroxylase activity. a) chitin group, b) chitosan group, c) control group.

inflammatory cells within the implants of the chitin and chitosan groups, but not in the control group. At Day 4, many inflammatory cells and fibroblasts were observed in the implants, while only a slight influx of these cells was observed in the control group. By Day 7, many implants of the chitin and chitosan groups were almost organized by granulation tissue (Fig. 2a, b), while the degree of organization of the implants varied widely in the control group (Fig. 2c). At Days 11 and 14, organization of the chitin and chitosan group implants was complete, while some of the control group implants showed incomplete organization. Many polykaryocytes were observed in the $10 \mathrm{mg}$ chitin group compared to the other groups at Day 7. At Day 11 and 14, 

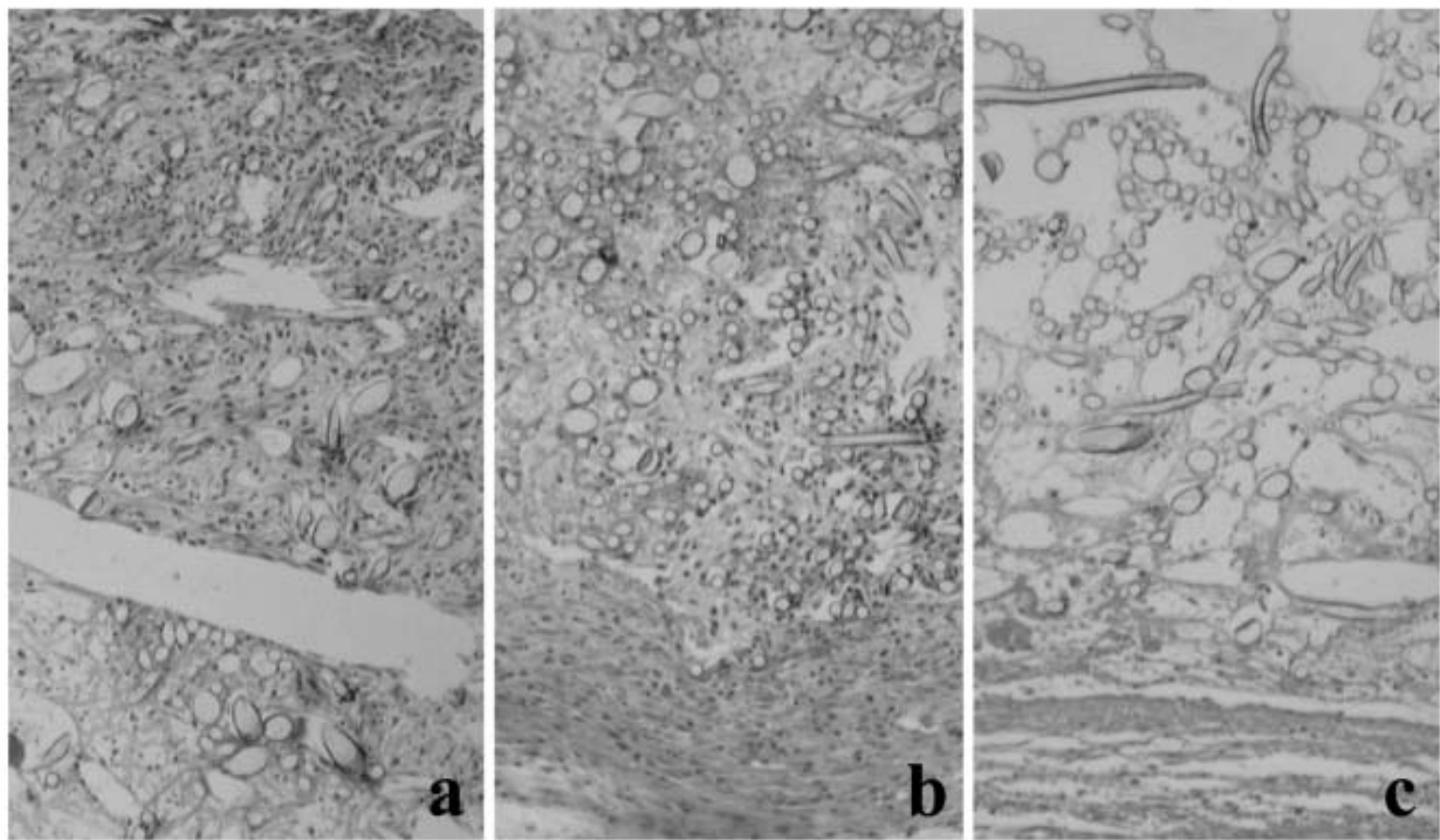

Fig. 2. Histological findings at 7 days post-implantation. a) $10 \mathrm{mg}$ chitin group, b) $1.0 \mathrm{mg}$ chitosan group, c) control group. The implants were almost organized by granulation tissue in the chitin and chitosan groups ( $a$ and $b$ ), while degree of organization $f$ the implant was less organized in the control group (c).

polykaryocytes were seen at the same level in all groups. There was no relationship between histological finding and the site impregnated in each group.

Chitin at the higher concentration was found to induce collagen synthesis without scatter in the early stages of wound healing in the present study. PHL is a necessary enzyme in the synthesis of collagen [18]. Thus, its activity can be considered an indicator of the degree of collagen synthesis. At Days 2 and 4, the activity of this enzyme was very low in every group. At this time, histological findings showed a prominent influx of inflammatory cells and a small number of fibroblasts. Therefore, it is speculated that the migration and proliferation of fibroblasts is not enough to promote collagen synthesis. At Day 7, the PHL activity increased in all samples, which indicates that collagen synthesis starts at this time. However, except for $10 \mathrm{mg}$ chitin group, scatter of the data varied widely in the other groups. In histological finding, organization of the implants was complete in the chitin and chitosan groups, while the degree of organization of the implants varied widely in the control group. Regarding $10 \mathrm{mg}$ chitin and control groups, histological findings were consistent with changes in the PHL activity. Pierce et al. reported that platelet derived growth factor (PDGF) stimulates the migration and proliferation of macrophages and fibroblasts [14]. Furthermore, PDGF activates the synthesis of transforming growth factor- $\beta$ (TGF- $\beta$ ) in macrophages, which also activates the synthesis of collagen in fibroblasts [14]. In addition, Shibata et al. indicated that chitin and chitosan activate macrophages [16]. We also observed histologically that polykaryocytes, which are thought to be formed by the fusion of macrophages, were induced by chitosan and more notably by chitin [12]. From present and previous reports [12], it is thought that chitin at higher concentration stimulates platelets and macrophages, resulting in the release of PDGF and TGF- $\beta$. In the present study, the NWF alone (control) was placed together with the NWF impregnated chitin and chitosan suspensions in each rat. However, polykaryocytes were seen frequently in the implants in the chitin and the chitosan groups, in particular in the $10 \mathrm{mg}$ chitin group. This phenomenon suggests that chitin and chitosan influence only locally, but not systemically.

At Day 14, PHL levels in the $1.0 \mathrm{mg}$ chitosan group were the highest among all groups. We previously reported that chitosan induces the formation of more granulation tissue in cats than chitin [4]. The present results support our previous data. In a similar study, Madden and Peacock reported that the PHL activity reached maximum at 14 days post-operation which was maintained until 10 weeks post-operation in a rat model [6]. In the present study, we investigated its activity until 14 days post-implantation. A prolonged examination would be useful.

\section{REFERENCES}

1. Allan, G.G., Altman, L.C., Bensinger, R.E., Ghosh, D.K., Hirabayasi, Y. and Neogi, S. 1984. pp. 119-133. In: Chitin, Chitosan and Related Enzymes (Zikakis J.P. ed.), Academic Press, Orland. 
2. Hutton, J. J., Tappel, A. L. Jr. and Udenfriend, S. 1966. Analy. Biochem. 16: 384-394.

3. Kishimoto, S. and Tamaki, K. 1987. Acta Dermato-Kyoto. 82: 471-479. (in Japanese).

4. Kojima, K., Okamoto, Y., Miyatake, K., Kitamura, Y. and Minami, S. 1998. Carbohydr. Polym. 37: 109-113.

5. Kojima, K., Okamoto, Y., Miyatake, K., Shigemasa, Y. and Minami, S. 2001. Carbohydr. Polym. 46: 235-239.

6. Madden, J. W. and Peacock, E. E. Jr. 1968. Surgery 64: 288294.

7. Minami, S., Okamoto, Y., Tanioka, S., Sashiwa, H., Saimoto, H., Matsuhashi, A. and Shigemasa, Y. 1993. pp. 141-152. In: Carbohydrates and Carbohydrate Polymers (Yalpani, M. ed.), ATL Press, Mount Prospect.

8. Minami, S., Okamoto, Y., Miyatake, K., Matsuhashi, A., Kitamura, Y., Tanigawa, T., Tanaka, Y. and Shigemasa, Y. 1996. Carbohydr. Polym. 29: 295-299.

9. Okamoto, Y., Minami, S., Matsuhashi, A., Sashiwa, H., Saimoto, H., Shigemasa, Y., Tanigawa, T., Tanaka, Y. and Tokura, S. 1993a. J. Vet. Med. Sci. 55: 739-742.

10. Okamoto, Y., Minami, S., Matsuhashi, A., Shigemasa, Y., Saimoto, H., Tanigawa, T., Tanaka, Y. and Tokura, S. 1993b. J. Vet. Med. Sci. 55: 743-747.
11. Okamoto, Y., Shibazaki, K., Minami, S., Matsuhashi, A., Tanioka, S. and Shigemasa, Y. 1995. J. Vet. Med. Sci. 57: 851854.

12. Okamoto, Y., Minami, S., Matsuhashi, A., Tanioka, S. and Shigemasa, Y. 1995. J. Jpn. Soc. Biomater. 13: 112-116.

13. Okamoto, Y., Southwood, L., Stashak, T. S., Norrdin, R. W., Nelson, A. W., Minami, S., Matsuhashi, A., Kato, K. and Shigemasa, Y. 1997. Carbohydr. Polym. 33: 33-38.

14. Pierce, G. f., Mustoe, T. A., Lingelbach, J., Masakowski, V. R., Gramates, P. and Deuel, T. F. 1989. Proc. Natl. Acd. Sci. U.S.A. 86: 2229-2233.

15. Prudden, J.F., Migel, P., Hanson, P., Friedlich, L. and Ballassa, L.L. 1970. Am. J. Surg. 119: 560-564.

16. Shibata, Y., Foster, L. A., Metzger, W. J. and Myrvik, Q. N. 1997. Infect. Immun. 65: 1734-1741.

17. Shigemasa, Y., Matsuura, H., Sashiwa, H. and Saimoto, H. 1996. Int. J. Biol. Macromol. 18: 237-242.

18. Shirai, M., Fujise, H., Masaoka, T., Takeda, S., Tsuboi, T. and Akahori, F. 1993. J. Toxicol. Sci. 18: 167-170.

19. Tokura, S. and Nishi, N. 1995. pp. 67-86. In: Chitin and Chitosan (Mat. B. Zaria, Wan Mohamed Wan Muda and Md. Paudi Abdullah eds.), Penerbit Universiti Kebangsaan, Malysia. 